\title{
Modelling of plasma rotation accounting for a poloidal divertor and helical perturbation coils
}

\author{
A. Nicolai ${ }^{1}$, U. Daybelge ${ }^{2}$ and C. Yarim ${ }^{2}$ \\ ${ }^{1}$ Institut für Plasmaphysik, Forschungszentrum Jülich $\mathrm{GmbH}$, Euratom Association, \\ Trilateral Euregio Cluster, D-52425 Jülich, Germany \\ 2 Istanbul Technical University, Faculty of Aeronautics and Astronautics, 80626 Maslak, \\ Istanbul, Turkey
}

Received 5 August 2005, accepted for publication 31 October 2005

Published 15 March 2006

Online at stacks.iop.org/NF/46/S145

\begin{abstract}
The impact of helical perturbations on the rotation velocity and thus on the energy confinement is calculated on the basis of the ambipolarity constraint, the parallel momentum equation of the revisited neoclassical theory and a simplified temperature equation. The helical perturbations can act as means for ergodizing the magnetic field and/or as momentum source or sinks, whereas at the separatrix (effective radius $r_{\mathrm{s}}$ ) of the poloidal divertor a temperature pedestal may arise due to the strong shear flow reducing the transport to a neoclassical level. The neoclassical theory allows the prediction of the parallel and poloidal flow speeds and thus of the 'subneoclassical' heat conductivity $\chi_{\text {sub }}$ used in the heat conduction equation. This heat conductivity allows us to compute the temperature pedestal and to reproduce the power balance in ALCATOR if one assumes that $\chi=\chi_{\text {sub }}$ in the radial sheath with the thickness of $\Delta \approx 0.7 \mathrm{~cm}$, centred around the inflection radius $r_{\text {in }}$, and $\chi=\chi_{\mathrm{L}}$ for $r<r_{\text {in }}-\Delta / 2 . \chi_{\mathrm{L}}$ is the normal L-mode heat conductivity.

Source terms account for momentum deposition by neutral beam injection (NBI), by pressure anisotropization and the $\vec{j} \times \vec{B}$ force density, the latter two due to Fourier components of (rotating) helical fields. Source terms for the power deposition by NBI, Ohmic heating and radiation are also included.

The main results can be summarized as follows:

At a dynamic ergodic divertor in TEXTOR frequency of $10 \mathrm{kHz}$, a toroidal velocity gradient of $1.2 \times 10^{6} \mathrm{~s}^{-1}$ may be achieved which is enough to suppress the ion temperature gradient and thus to generate an ITB.

The poloidal divertor suppresses the neutral gas influx and thus effects a (sub)neoclassical sheath with a temperature pedestal of $T_{\text {ped }} \approx 400 \mathrm{eV}$ and an increase of the central value by roughly the same amount. In the case of edge localized mode-control with an ergodic layer of $\Delta \approx 2.5 \mathrm{~cm}$, generated by the helical coils, the height of the pedestal stays unaffected if in the pedestal region a transition from L-mode confinement to subneoclassical confinement is assumed.
\end{abstract}

PACS numbers: 52.55.Fa, 52.30.- $\mathrm{q}$

(Some figures in this article are in colour only in the electronic version)

\section{Introduction}

Anomalous plasma transport and the concomitant deterioration of the confinement times of tokamaks much below the neoclassical prediction, in particular if auxiliary heating is applied, are key issues in fusion research.

Therefore the surprising experimental discovery of the transition to a high confinement mode, in particular in divertor plasmas, has evoked considerable interest in improved confinement regimes, which seem to be related to toroidal and/or poloidal plasma spin up and the rise of (sheared) radial electric fields [1-3].

The revisited neoclassical theory [2-7], which is based on Braginskii's equations [8,9], allows within the framework of a rigorous analytical approach the calculation of the two dimensional velocity field on the flux surfaces and the perpendicular ambipolar electric field, i.e. the quantities which are believed to be important for the $\mathrm{L}-\mathrm{H}$ transition.

The theory is valid in collision dominated plasmas with steep gradients and was able to reproduce the 
toroidal spin up in the divertor tokamak ALCATOR C-MOD $[4,5]$.

The importance of the theory is underlined by the fact that an interaction between the poloidal or toroidal spin up and the turbulence-driven anomalous transport is believed to be a likely reason for the aforementioned $\mathrm{L}-\mathrm{H}$ mode transition in tokamaks.

It is also worthwhile to mention that ignition of the International Tokamak Experimental Reactor (ITER) requires an improved confinement regime.

However, the large pressure gradient in the pedestal region gives rise to the edge localized modes (ELMs) which are supposed to be dangerous for the divertor plate and therefore should be mitigated by ergodizing the edge region inside the separatrix [15]. The ergodization leads to increased transport above the (sub)neoclassical level and to a reduction of the pressure gradient below the ballooning-peeling limit.

In [12] a consistent modelling of the momentum transport was aimed at which accounts for anomalous viscosity, neutral beam injection (NBI), friction due to recycled neutrals, braking due to pressure anisotropization and the momentum source due to $\vec{j} \times \vec{B}$ force density in the singular layer, the latter two evoked by the Fourier components of external nonaxisymmetric fields.

Here also a simplified heat conduction equation accounting for Ohmic heating, deposition by NBI and radiation is considered to compute the temperature pedestal due to the poloidal divertor.

To account for the turbulence prevailing, e.g. in the L-mode of TEXTOR, the possibility is foreseen of introducing an anomalous viscosity [10]. Since the ion temperature gradient (ITG) threshold is exceeded in the TEXTOR L-mode, this instability, together with the trapped electron mode, are considered here as the main source of turbulence.

The friction with the recycled neutral gas due to charge exchange is, in limiter devices, a strong source of momentum loss, as pointed out in [4]. In the divertor case the recycling zone is shifted partly in front of the plate and the neutral influx is strongly reduced.

The dominating friction of the rotating plasma with embedded islands generated by a radial magnetic field has been already discussed in [11]. The radial magnetic field is assumed there to be static. Here the case of a rotating radial field is envisaged as well.

In particular, at TEXTOR and JET, static or rotating helical fields will be applied to influence the plasma rotation driven mainly by NBI. Here an attempt is made to describe these experiments numerically.

The paper is organized as follows. In section 2 the main results of the revisited neoclassical theory are summarized and the characteristic quantities are given. Then a somewhat simplified power balance (section 3 ) is presented and a transport model accounting for rotation and heat conduction is set up. The momentum sources are discussed in section 4 . Finally, results are presented in section 5.

\section{Ambipolarity constraint and parallel momentum balance}

As mentioned already, the revisited neoclassical theory accounts in particular for steep temperature and density

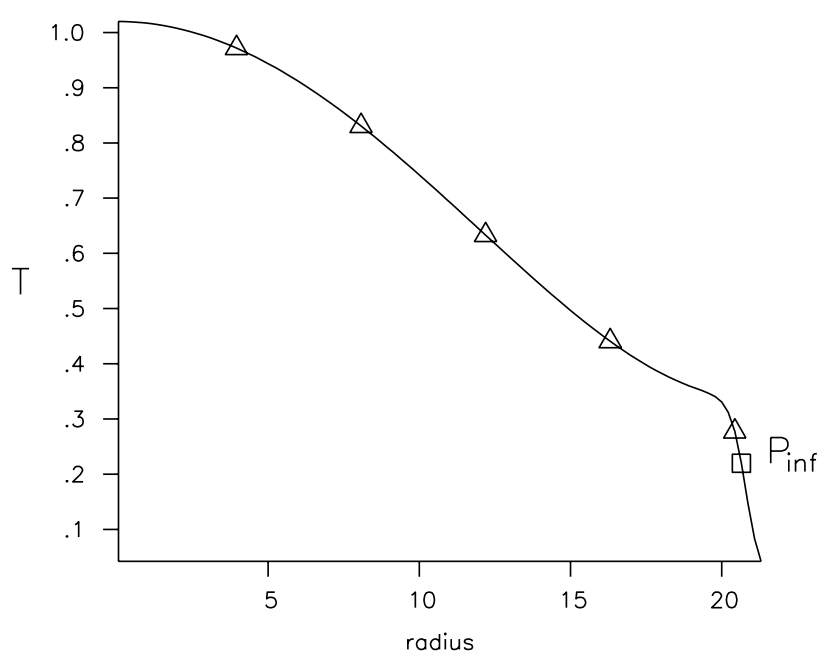

Figure 1. Ion temperature $T_{\mathrm{i}}[\mathrm{keV}]$ (ALCATOR) versus radius $r[\mathrm{~cm}]$. The maximum ion temperature is $2.1 \mathrm{keV}$. The temperature pedestal has the inflection point $P_{\text {inf }}$.

gradients at high collisionality. Since it is a collision dominated theory, it is based on the fluid equations for particle, momentum and energy conservation [2-7]. Accounting for parallel, perpendicular and gyro-viscosity $[8,9]$, we get from the ambipolarity constraint [12]

$$
\begin{gathered}
\frac{1}{r} \frac{\partial}{\partial r}\left[r \eta_{2}\left(\frac{\partial u_{\phi}}{\partial r}-M_{1} \frac{0.107 q^{2}}{1+\left(Q^{2} / S^{2}\right)} \frac{\partial \ln T}{\partial r} \frac{B_{\phi}}{B_{\theta}} u_{\theta}\right)\right] \\
=m_{\mathrm{i}} n_{\mathrm{i}}\left(\frac{\partial}{\partial t}+v_{\mathrm{cx}}\right) u_{\phi}+T_{\mathrm{NBI}}+T_{\mathrm{ANI}}+T_{j \times B} .
\end{gathered}
$$

Here $u_{\theta}$ is the averaged poloidal speed, $\eta_{2}$ is the perpendicular viscosity coefficient and $M_{1}$ is a metric quantity of the order unity accounting for elongated cross-sections $[4,12]$. We note that as in [12] the classical viscosity must be replaced by an anomalous one to reproduce TEXTOR —or JET-data. The velocities $Q$ and $S$ are defined in [4,12] as well. The charge exchange reactions are characterized by the charge exchange frequency $v_{\mathrm{cx}}=\langle\sigma v\rangle_{\mathrm{cx}} n_{0} .\langle\sigma v\rangle_{\mathrm{cx}}$ is the rate coefficient for charge exchange and $n_{0}$ the neutral gas density. The source terms $T_{\mathrm{NBI}}, T_{\mathrm{ANI}}$ and $T_{j \times B}$ account for NBI, pressure anisotropization due to helical perturbations and for the $\vec{j} \times \vec{B}$ force at the singular surfaces [12]. We define the dimensionless quantities $g=u_{\phi} / v_{T}, h=u_{\theta} / v_{T}$ and $x=\left(r-r_{\text {in }}\right) / L_{\psi}$. Here we have used the (constant, positive) velocity $v_{T}=$ $\left(1 / e B_{\phi}\right)\left(T_{\text {in }} / L_{\psi}\right) . T_{\text {in }}$ is the temperature at the point $P_{\text {in }}$ with radius $r_{\text {in }}$ and the length $L_{\psi}=L_{T}\left(r_{\text {in }}\right)$ is $L_{T}$ at $P_{\text {in }}$. In the case of ALCATOR C-MOD [4] $P_{\text {in }}$ is the 'inflection' point $P_{\text {inf }}$ which is defined in figure 1 as the locus of vanishing curvature of the ion temperature profile. In the case of TEXTOR (without temperature pedestal) $P_{\text {in }}$ is assumed to coincide with the plasma edge (i.e. $r_{\text {in }}=r_{\mathrm{s}}, r_{\mathrm{s}}$ is the minor plasma radius). The length $L_{\psi}=L_{T}\left(r_{\text {in }}\right)$ is $L_{T}=(\partial \ln T / \partial r)^{-1}$ at $P_{\text {in }}$. We get the dimensionless equation $\left(x^{\prime}=x+\left(r_{\text {in }} / L_{\psi}\right)\right)$

$$
\frac{1}{x^{\prime}} \frac{1}{\eta_{2}} \frac{\partial}{\partial x}\left[x^{\prime} \eta_{2} G\right]=\hat{T}_{\mathrm{cx}}+\hat{T}_{\mathrm{NBI}}+\hat{T}_{\mathrm{ANI}}+\hat{T}_{j \times B} .
$$

The quantity $G$ is given by

$$
G=\frac{\partial g}{\partial x}-\frac{0.107 q^{2}}{1+\left(Q^{2} / S^{2}\right)} \frac{\partial \ln T}{\partial x} \frac{B_{\phi}}{B_{\theta}} h .
$$


The second term in equation (3) acts as a source term which is roughly proportional to $(\partial \ln T / \partial x) h$, i.e. to the temperature gradient. $q$ is the safety factor and $R$ the major radius. The dimensionless source term $\hat{T}_{\mathrm{cx}}$ is $\hat{T}_{\mathrm{cx}}=t_{\mathrm{c}, \text { in }}\left(\hat{n} / \hat{\eta}_{2}\right) v_{\mathrm{cx}} g$. It accounts for the aforementioned friction evoked by the neutral gas. The characteristic time $t_{\mathrm{c}, \text { in }}$ at the point $P_{\text {in }}$ is given by $t_{\mathrm{c}, \text { in }}^{-1}=\left(1 / m_{j} n_{\mathrm{i}} L_{\psi}^{2}\right) \eta_{2 \text {,in }}$. The 'relative' viscosity $\hat{\eta}_{2}$ is defined by $\eta_{2}=\hat{\eta}_{2} \eta_{2, \text { in }} . \eta_{2 \text {,in }}$ is the viscosity at the point $P_{\text {in }}$. Due to the scaling of $\tau_{\mathrm{i}}$ with density and temperature we get $\hat{\eta}_{2}=\hat{n}^{2} \hat{T}^{-(1 / 2)+Z} . \hat{T}=\left(T / T_{\text {in }}\right)$ is the relative temperature and $\hat{n}=\left(n / n_{\text {in }}\right)$ the relative density. $T_{\text {in }}$ and $n_{\text {in }}$ are the temperature and density at the point $P_{\text {in. }}$. As temperature dependence of $Z_{\text {eff }}$ the relation $Z_{\text {eff }}=Z_{\text {eff }} \hat{T}^{Z}$ is assumed, $\mathrm{Z}$ is a constant.

Equation (2) is a second order equation for the normalized toroidal velocity $g$.

Using the rate coefficient for charge exchange with cold neutrals $(\approx 5 \mathrm{eV})\langle\sigma v\rangle_{\mathrm{cx}}=4.7 \times 10^{-14} \mathrm{~m}^{3} \mathrm{~s}^{-1}$, the charge exchange frequency of a neutral gas with the density $n_{0}\left(10^{19} \mathrm{~m}^{-3}\right)$ is given by $v_{\mathrm{cx}}=4.7 \times 10^{5} \cdot n_{0}$. We assume that the neutral gas density decays as $n_{0}=N_{0} \exp \left(L_{\psi} / L_{\text {neu }} x\right) . N_{0}$ is the neutral density at the inflection point. $L_{\text {neu }} \approx 1 \mathrm{~cm}$ is an estimate of the decay length indicated by the SPUDNUT code [14], assuming deuterium atoms only.

Taking the parallel components of the terms in the momentum equation [12] and accounting in particular for the gyro-viscosity tensor [4] we get in the case of large aspect ratio and circular cross-section a nonlinear relation between the poloidal and toroidal plasma velocities [4].

$$
\begin{aligned}
\left(h^{*}+\right. & 1.833) M_{1} \gamma=\frac{0.45 \Lambda}{1+\left(Q^{2} / S^{2}\right)}\left\{\frac{0.107 q^{2}}{1+\left(Q^{2} / S^{2}\right)} h^{*}\right. \\
& +\frac{1}{2} \frac{1}{[\partial \hat{T} / \partial x]^{2}}\left(g^{*}\right)^{2}-\frac{1}{\partial \hat{T} / \partial x} g^{*}\left[h^{*}-\left(1+\frac{2}{\eta}\right)\right] \\
& \left.+1.9\left[h^{*}-0.8\left(1+\frac{1.6}{\eta}\right)\right]^{2}\right\} .
\end{aligned}
$$

Here the definitions $g^{*}=\left(B_{\theta} / B_{\phi}\right)\left(u_{\phi} / \eta v_{n}\right)$ and $h^{*}=u_{\theta} / \eta v_{n}$ are used, to cast equation (4) in a convenient form. The important quantity $\Lambda=\left(v_{\mathrm{i}} / \Omega_{\mathrm{i}}\right)\left(q^{2} R^{2} / L_{T} r\right)$ acts as a switch for the finite Larmorradius effects. For large gradient lengths $L_{T}$ the neoclassical result is retrieved. $M_{1}$ and $\gamma$ depend on the flux surface geometry. We introduce the effective radius [12], and use $M_{1}=1$ as in equation (1) and $\gamma=1$ as in [21].

\section{Power balance}

As (somewhat simplified) power balance equation for the transport perpendicular to the flux surfaces we use

$$
\begin{gathered}
\frac{3}{2} \frac{\partial}{\partial t}\left(n\left(1+R_{n}\right) k T\right)=-\frac{1}{r} \frac{\partial}{\partial r}\left[-\left(R_{n} \chi_{\mathrm{e}}+\chi_{\mathrm{i}}\right) n \frac{\partial T}{\partial r}\right. \\
\left.+C_{\mathrm{V}}\left(T_{\mathrm{e}} \Gamma_{\mathrm{e}}+T_{\mathrm{i}} \Gamma_{\mathrm{i}}\right)\right]+P_{\mathrm{OH}}+P_{\mathrm{inj}}+P_{\mathrm{rad}} .
\end{gathered}
$$

The purpose of this equation is to show the effect of a reduction of $\chi$ to a neoclassical level because of an increased velocity shear. To be consistent with the assumption of high density we assume $T_{\mathrm{i}}=T_{\mathrm{e}}=T$. In addition we use $R_{n}=n_{\mathrm{e}} / n \approx 1$. $P_{\mathrm{OH}}$ is the Ohmic heating power density, $P_{\text {inj }}$ the power density deposited by NBI and $P_{\text {rad }}$ the power density radiated away. Figure 2 [6] shows the deposition profile of the radiation

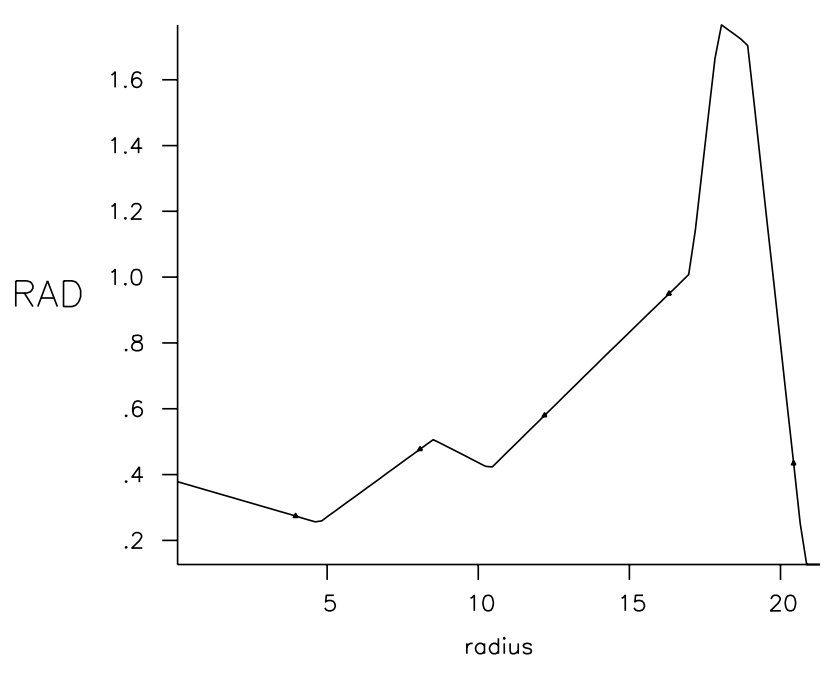

Figure 2. Distribution of the radiation losses $\left[\mathrm{W} \mathrm{m}^{-3}\right]$ in ALCATOR versus radius $r[\mathrm{~cm}]$. The same distribution, however renormalized, is used for TEXTOR.

losses in ALCATOR. Since in the case of low-Z impurities the radiation losses are peaked in the vicinity of the edge (as in figure 2), this profile is used for other cases as well, however, with a changed normalization, to meet the total radiation losses.

We assume that the convective part due to radial diffusion is small compared with the conduction part $\left(C_{\mathrm{V}}=0\right)$. The total Ohmic power is $P_{\Omega}=\int 2 \pi^{2} R_{0} P_{\mathrm{OH}} r \mathrm{~d} r=U I_{\mathrm{p}} . U$ is the loop voltage and $I_{\mathrm{p}}$ the plasma current. As power density we take $P_{\mathrm{OH}}=\alpha_{\mathrm{dep}} \eta_{\mathrm{sp}} j_{\mathrm{z}}^{2}=\alpha_{\mathrm{dep}}\left[U^{2} /\left(2 \pi R_{O}\right)^{2} \eta_{\mathrm{sp}}\right]$ where $j_{\mathrm{z}}$ is the toroidal current density and $\eta_{\mathrm{sp}}$ the Spitzer-resistivity (in MKSA units): $\eta_{\mathrm{sp}}=1.61 \times 10^{-9} \lambda_{\text {coul }}\left(\mathrm{keV} / k T_{\mathrm{e}}\right)^{3 / 2} Z_{\text {eff }} \Omega m$, $\lambda_{\text {coul }}$ is the Coulomb-logarithm. The prefactor $\alpha_{\text {dep }}$, accounting for trapping and the impurities, is determined from the equation for the total Ohmic power. We get $\alpha_{\text {dep }}=\left(R P_{\Omega} / U^{2}\right)$ with $(1 / R)=\left(1 / 2 \pi R_{0}\right) \int\left(\mathrm{d} f / \eta_{\mathrm{sp}}\right), \mathrm{d} f=2 \pi r \mathrm{~d} r$. As in equations (2) and (4) we concentrate on the stationary equation $(\partial / \partial t=0)$.

Three approaches with the heat diffusivities $\chi_{\text {tot }_{1-3}}$ were used in an attempt to reproduce the temperature profile in ALCATOR C-MOD and to predict the temperature profile in a medium size divertor device:

(1) A parabolic dependence is used in $0<r<r_{\text {in }}-\Delta / 2$ and subneoclassical behaviour in the pedestal (with a width $\Delta \approx L_{\psi}$ and the inflection radius $r_{\mathrm{in}}: \chi_{\mathrm{tot}_{1}}=(1+$ $\left.\gamma\left(r^{2} / r_{\text {in }}^{2}\right)\right) \chi_{\text {anom }} S_{\text {off }}+\chi_{\text {neo }} S_{\text {on }} . S_{\text {off }}, S_{\text {on }}$ and $\chi_{\text {neo }}$ are given by $S_{\text {off }}=1 /\left(1+\exp \left(r-r_{\text {in }}-(\Delta / 2) / \delta_{\text {trans }}\right)\right), S_{\text {on }}=1-$ $S_{\text {off }}$ and $\chi_{\text {neo }}=\kappa_{0}\left[1+1.6\left(q^{2} / 1+\left(Q^{2} / S^{2}\right)\right)\right] v_{\mathrm{i}} r_{\mathrm{ci}}^{2}=\kappa_{0}[1+$ $\left.1.6\left(q^{2} / 1+\left(Q^{2} / S^{2}\right)\right)\right] v_{\mathrm{i}}\left(2 k T_{\mathrm{i}} / m_{\mathrm{i}} \Omega_{\mathrm{i}}^{2}\right)$, respectively. Thus $S_{\text {on }}$ is a smeared out step function with the centrepoint just at the inner edge of the pedestal $\left(r=r_{\text {in }}-\Delta / 2\right)$. The subneoclassical heat diffusivity also contains the dependence on plasma rotation via $Q$ and $S$ [2]. Except if it is stated otherwise, the transition length $\delta_{\text {trans }}=1 \mathrm{~mm}$ is chosen to be small compared with $\Delta$.

(2) In $0<r<r_{\text {in }}-\Delta / 2$ the coefficient obtained by inverting the temperature profile and inside the pedestal $r_{\text {in }}-\Delta / 2<r<r_{\text {in }}+\Delta / 2$ the subneoclassical dependence is used. Thus we have $\chi_{\text {tot }}=\chi_{\text {tot }_{2}}=\chi_{\text {inv }} S_{\text {off }}+\chi_{\text {neo }} S_{\text {on }}$. 


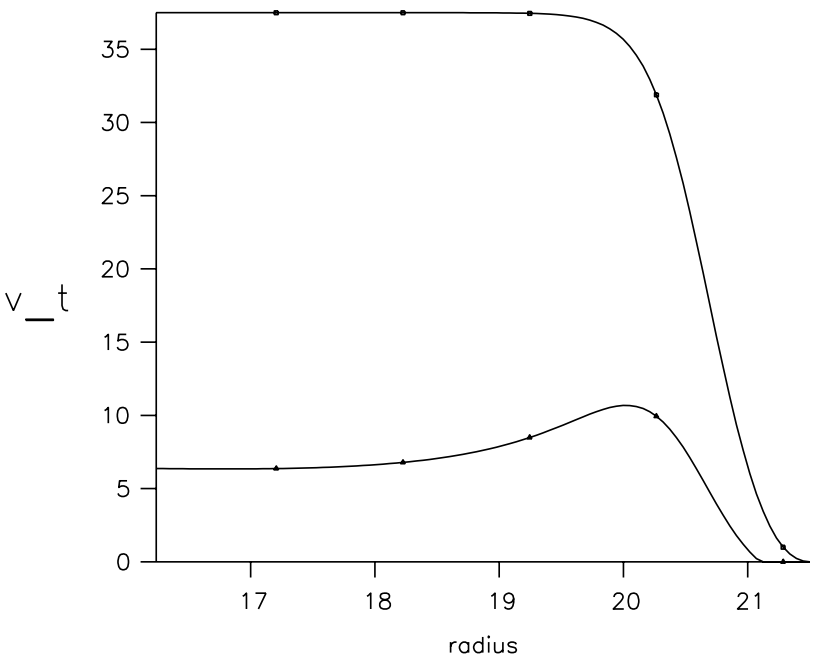

Figure 3. Toroidal speed $v_{\phi}\left[\mathrm{km} \mathrm{s}^{-1}\right]$ (ALCATOR) versus radius $r[\mathrm{~cm}]$. The lower curve belongs to $n_{0}=5 \times 10^{15} \mathrm{~cm}^{-3}$, the upper to $n_{0}=0$ thus demonstrating the influence of the neutral gas.

(3) The coefficient obtained by inverting the temperature profile is used everywhere: $\chi_{\mathrm{tot}_{3}}=\chi_{\text {inv }}$. This reproduces the original profile (used as a check). Equations (2) and (5) are second order equations which are decomposed in four first order equations that are solved by a standard shooting method.

\section{Momentum sources}

The momentum sources due to NBI, due to pressure anisotropization and due to the $\vec{j} \times \vec{B}$-forces were discussed in detail in [12]. Here we concentrate on the influence of the neutral gas which is important for the action of the poloidal divertor giving rise to a temperature pedestal due to a transport barrier at the edge. Also the influence of a rotating helical field, which may accelerate the rotating plasma and produce an ITB in the interior, is discussed.

The toroidal velocity of figure 3 was obtained by assuming $n_{0}=0$. In figure $7 n_{0}=5 \times 10^{15} \mathrm{~m}^{-3}$ was assumed; this leads to a reduction of the central velocity by $60 \%$. This density corresponds (presupposing ALCATOR geometry) to a particle confinement time $\tau_{\mathrm{p}}=100 \mathrm{~m} \mathrm{~s}$, a mean density $\langle n\rangle=10^{20} \mathrm{~m}^{-3}$ and mean kinetic energy of the recycled neutral gas of $5 \mathrm{eV}$. Thus a reduction of the neutral gas seems to be a prerogative for an increased rotation which in turn reduces the edge confinement to the (sub)neoclassical. By concentrating the recycling to the vicinity of the target plate of the divertor the neutral gas density inside the separatrix is in fact strongly reduced. We note that a (more realistic) particle confinement time $\tau_{\mathrm{p}}=10 \mathrm{~m} \mathrm{~s}$ means $n_{0}=5 \times 10^{16} \mathrm{~m}^{-3}$ which stops the toroidal rotation.

\section{Results}

The aim is to investigate the build up of transport barriers.

The results concerning a possible transport barrier in the plasma interior due to an increased velocity shear in the case of a prescribed temperature profile (equations (2) and (4)) will be presented first.
The extended model based on equations (2), (4) and (5) was benchmarked with ALCATOR C-MOD data in [22] already. Here the main results are summarized.

In a second step the model is then applied to a medium size divertor-tokamak aiming at the (sub)neoclassical sheath at the edge which gives rise to a temperature pedestal. The resonant perturbation coil system as used at TEXTOR is then applied to elucidate the possibility of ELM mitigation which is an important issue for JET and ITER. We note that at DIII-D and JET mainly nonresonant configurations ('C-coils, I-coils') had been investigated [23].

\subsection{ITB due to plasma rotation}

The input data are those of TEXTOR. They are given by (shot \# 91269): $r_{\mathrm{in}}=r_{\mathrm{s}}=46 \mathrm{~cm}, R=175 \mathrm{~cm}, T_{\mathrm{i}_{\max }}=1500 \mathrm{eV}$, $T_{\mathrm{e}_{\max }}=1200 \mathrm{eV}, n_{\max }=5.4 \times 10^{13} \mathrm{~cm}^{-3}, \eta=1.6$ and $B_{\phi}=2.23 \mathrm{~T}$. NBI is characterized by $P_{\mathrm{MW}}=0.72, E_{\mathrm{keV}}=40$ (deuterons). The plasma current is $I_{\mathrm{p}}=350 \mathrm{kA}$ and the maximum toroidal speed $v_{\mathrm{t}_{\max }}=110 \mathrm{~km} \mathrm{~s}^{-1}$.

Figure 4 shows the temperature profile. The radii of the gridpoints of the abscissa axis are given in centimetres. This applies also for figures 5 and 6, 9-13 and 15 .

The maximum of the toroidal velocity (figure 5) is reproduced with an accuracy of around 10\%. A similar result is obtained for JET [12].

It is assumed that during the accelerating process the slip frequency [12] is below the critical value. This can be achieved by adjusting the rotating frequency of the helical field.

By gradually increasing the rotation speed of the helical field $\Omega_{\mathrm{f}}$, i.e. by keeping the slip frequency below the critical frequency [12], the maxima can be pushed to the point corresponding to the dynamic ergodic divertor (DED) frequency of $10 \mathrm{kHz}$ (figure 6). The gradient $\mathrm{d} v_{t} / \mathrm{d} r$ at $r=$ $40 \mathrm{~cm}$ is $1.2 \times 10^{6} \mathrm{~s}^{-1}$.

We note that the plasma acceleration shown in figure 6 entails a strong increase of the central velocity, by around a factor of two.

The stepwise increase of the frequency is achieved (numerically) by starting step $n$ by the boundary condition of step $(n-1)$. Around ten steps are needed.

For the suppression of the ITG-instability approximately a shearing rate $\omega_{E \times B} \approx\left(B_{\theta} / B\right)(\mathrm{d} / \mathrm{d} r) v_{\phi}>\gamma_{\mathrm{ITG}}$ is needed. Since according to [10] the growth rate is $\gamma_{\text {itg }} \approx 10^{5} \mathrm{~s}^{-1}$, the velocity gradient $\mathrm{d} v_{\phi} / \mathrm{d} r \approx 10^{6} \mathrm{~s}^{-1}$ is required. Therefore the achieved velocity gradient is sufficient-quite in contrast to the plasma breaking discussed in [12].

The (revolving) magnetic field structure of the DED may be characterized by the Fourier spectra [12]. Figure 7 shows the spectrum at the $q=2$ surface for the $(m=3, n=1)$ configuration $\left(I_{\mathrm{d}}=10 \mathrm{kA}\right)$, and figure 8 shows the spectrum at the $q=2$ surface for the ( $m=6, n=2)$ configuration $\left(I_{\mathrm{d}}=10 \mathrm{kA}\right)$.

\subsection{Generation of an edge transport barrier in a rotating plasma by means of a poloidal divertor and ELM-stabilization}

We treat the plasma rotation in divertor tokamaks which leads to a (sub)neoclassical sheath in the edge due to the large velocity shear $\left(\approx 5 \times 10^{6} \mathrm{~s}^{-1}\right)$. Important is the screening of the 


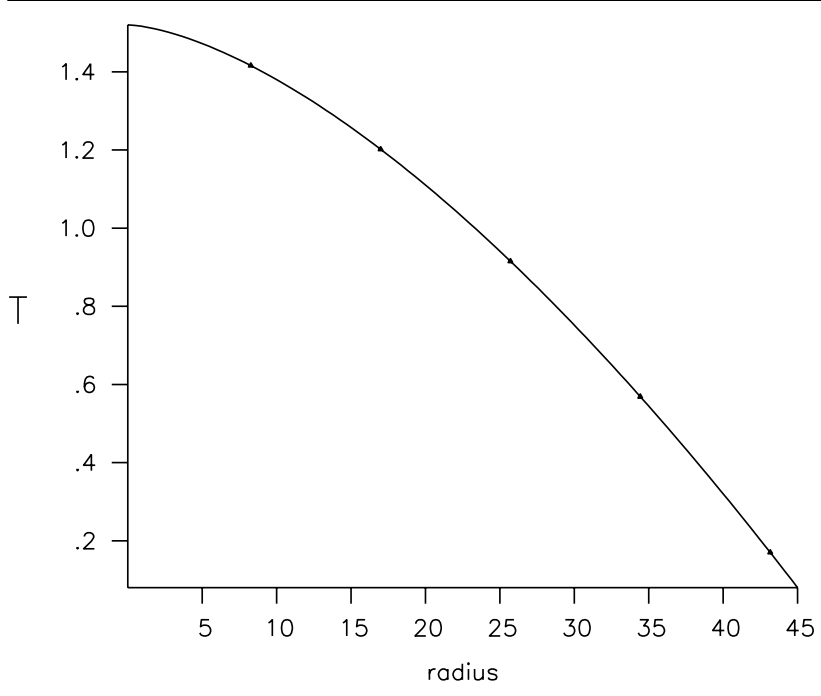

Figure 4. Ion temperature $T_{\mathrm{i}}[\mathrm{keV}]$ in TEXTOR versus radius $r$ $[\mathrm{cm}]$. The shape of the profile is similar to that of the toroidal velocity.

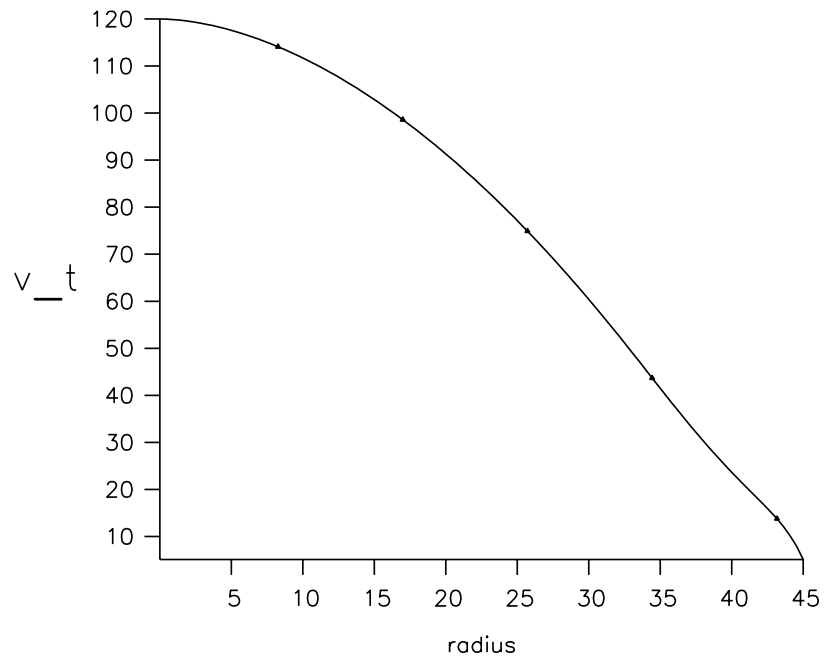

Figure 5. Toroidal speed $v_{\phi}\left[\mathrm{km} \mathrm{s}^{-1}\right]$ versus radius $r$ [cm]. The model reproduces the maximum velocity within an accuracy of $10 \%$.

neutrals. The strong magnetic shear at the separatrix, which may be important as well, enters via the $q$-profile.

The main results concerning the comparison with ALCATOR C-MOD are [4, 22]:

The observed maximum toroidal velocity $u_{\phi}=35 \mathrm{~km} \mathrm{~s}^{-1}$ (figure 3, upper curve) was reproduced with an accuracy of $10 \%$. The temperature profile in figure 1 was used as an input. Analytically, the profile is given by

$$
\begin{aligned}
T= & T_{\text {in }}\left\{\left(1-\tan h\left(\frac{r-r_{\text {in }}}{L_{\psi}}\right)\right)+\left(\frac{T_{0}}{T_{\text {in }}}-2\right)\right. \\
& \left.\times\left(1-\left[\frac{r}{r_{\text {in }}}\right]^{2}\right)^{2}\right\} .
\end{aligned}
$$

Here the profile parameters are given by $T_{0}=1020 \mathrm{eV}$, $T_{\text {in }}=165 \mathrm{eV}, r_{\text {in }}=20.8 \mathrm{~cm}$ and $L_{\psi}=0.7 \mathrm{~cm}$.

We use the heat conduction equation (5) to reproduce the temperature profile of figure 1 . The radial dependence in the case of the heat diffusivity $\chi_{\text {tot }_{1}}$ (equation (3)) is inaccurate

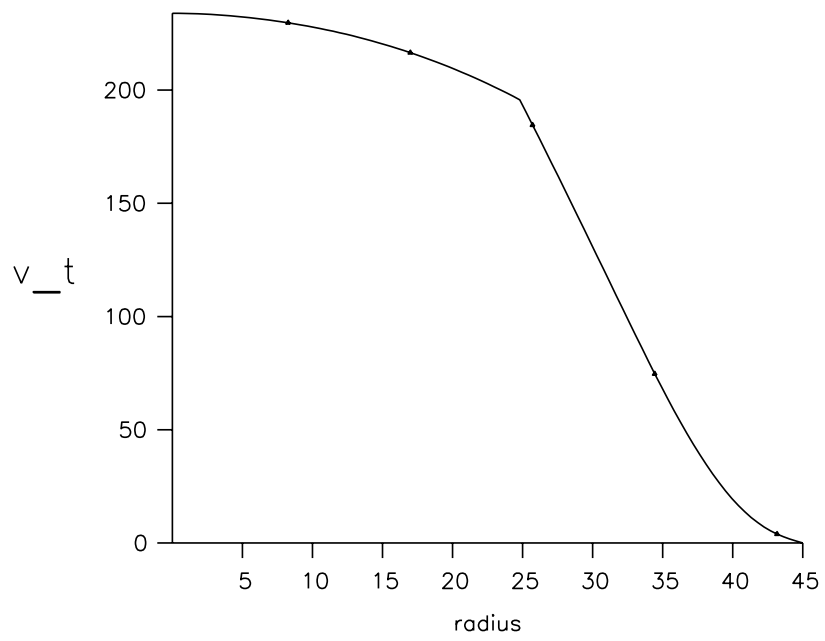

Figure 6. Toroidal speed $v_{\phi}\left[\mathrm{km} \mathrm{s}^{-1}\right]$ with helical field (TEXTOR) versus radius $r[\mathrm{~cm}]$. Accelerating by means of the $(m=2$, $n=1$ ) - Fourier component of the DED—field leads to a localized maximum at $r(q=2)=25 \mathrm{~cm}$ and an increase of the maximum speed to $230 \mathrm{~km} \mathrm{~s}^{-1}$.

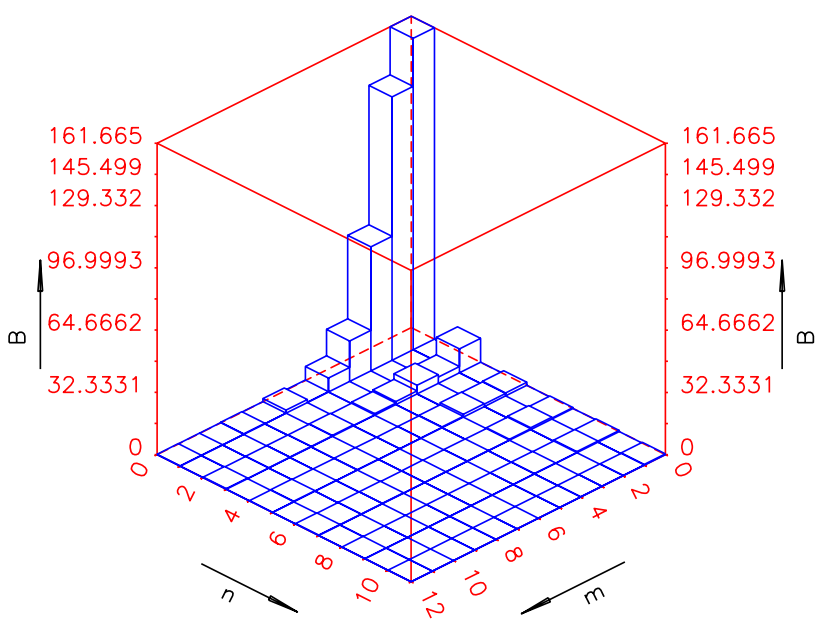

Figure 7. Spectrum of DED for $p_{3}=1$ (3/1 configuration). Due to the large distance between the $q=2$ surface and the winding the $(m=1, n=1)$-and $(m=2, n=1)$-components are larger than the $(m=3, n=1)$ components.

in approaching the pedestal (figure 9). This improves considerably in using the diffusivity $\chi_{\text {tot }_{2}}$ (equation (3) with $\kappa_{0}=1$ (figure 10). The total heat diffusivity $\chi_{\mathrm{tot}_{3}}=\chi_{\mathrm{inv}}$ may be compared with the neoclassical and subneoclassical one (figure 11). In the gradient region $(15 \mathrm{~cm}<r<20 \mathrm{~cm}$ ) $\chi_{\text {inv }}$ is much larger than the neoclassical ion heat diffusivity. However, in the vicinity of the inflection point $\chi_{\text {inv }}$ lies in between the neoclassical and the subneoclassical diffusivity (it is roughly the mean value of both). This clearly shows that in this region $\chi_{\text {inv }}<\chi_{\text {neo }}$ holds. Figure 17 shows the poloidal divertor configuration envisaged in this numerical study. The axisymmetric coils are located (without loss of generality concerning the considerations here) at the inboard side at $\theta=225^{\circ}$ and at $\theta=135^{\circ}$. The divertor current is $I_{\text {div }}=100 \mathrm{kA}$.

We assume that the neutral gas is recycled in the divertor plasma without entering the main plasma chamber. The complicated neutral gas and plasma transport physics is not 


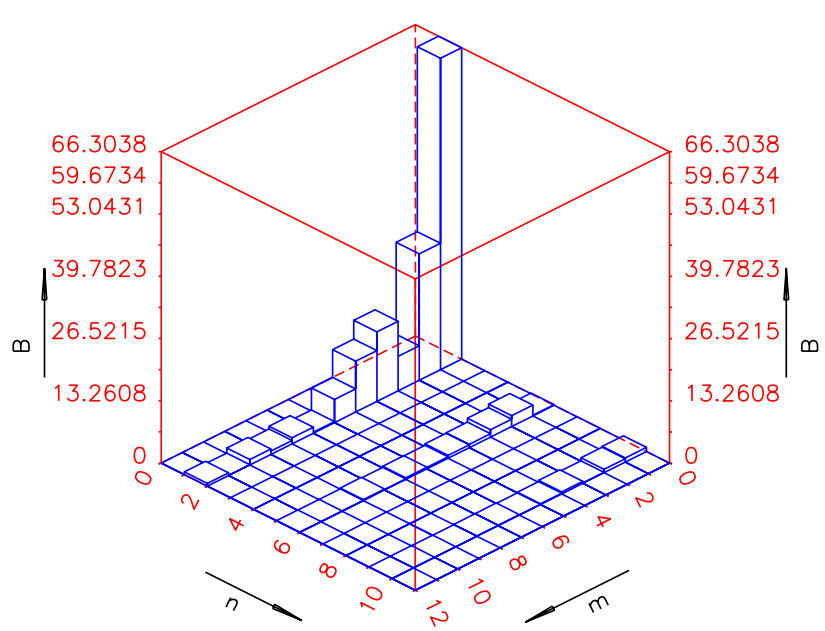

Figure 8. Spectrum of DED for $p_{3}=2$ (6/2-configuration). Due to the large distance between the $q=2$ surface and the winding the $(m=1, n=1)$-and $(m=2, n=1)-$ components are larger than the $(m<6, n=1)$ components.

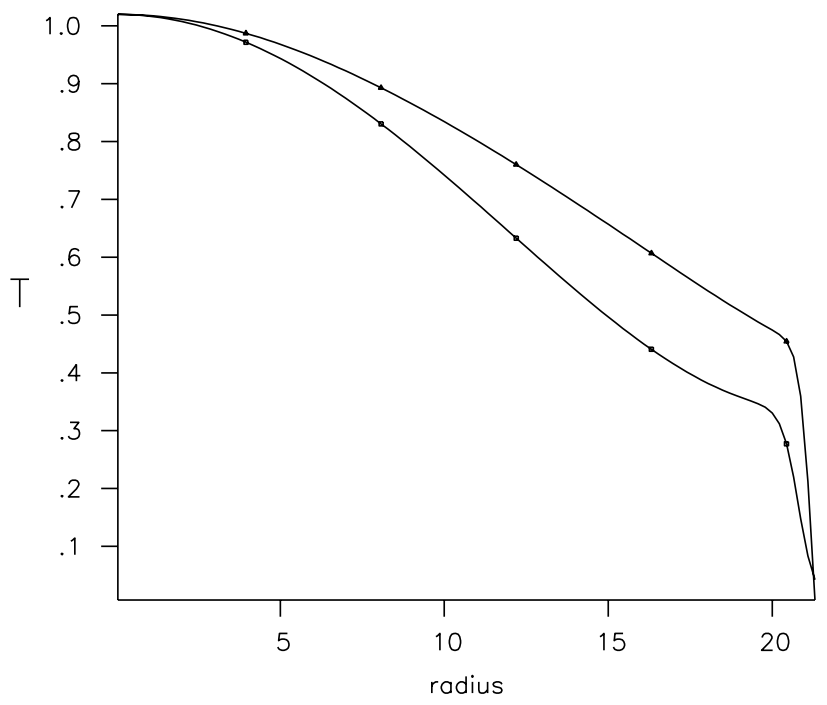

Figure 9. Ion temperature in ALCATOR [keV] versus radius $r$ $[\mathrm{cm}]$. The lower curve is the experimental one and the upper belongs to the coefficient in equation (3) thus demonstrating the rough approximation.

assessed here. The position of the X-points does not enter in equations (2)-(5). Therefore the particular choice is unimportant and the considerations are approximately valid for devices with roughly the same effective radius, major radius and toroidal field as given above. The assumptions for the simulation are as follows:

(1) We have subneoclassical transport with $\kappa_{0}=1$ inside the pedestal $r_{\text {in }}-\Delta<r<r_{\text {in }}+\Delta$.

(2) Outside the pedestal (in $0<r<r_{\text {in }}-\Delta$ ) the temperature profile in figure 4 is inverted and the obtained heat diffusivity is used in equation (5). Thus it is assumed that the L-mode heat diffusivity is also valid in the H-mode except in the edge where the barrier due to the shear flow is build up. Since the height of the pedestal is around $400 \mathrm{eV}$ transport regimes other than in the L-mode may be entered; however, to apply a multiregime transport model is beyond the scope of this contribution.

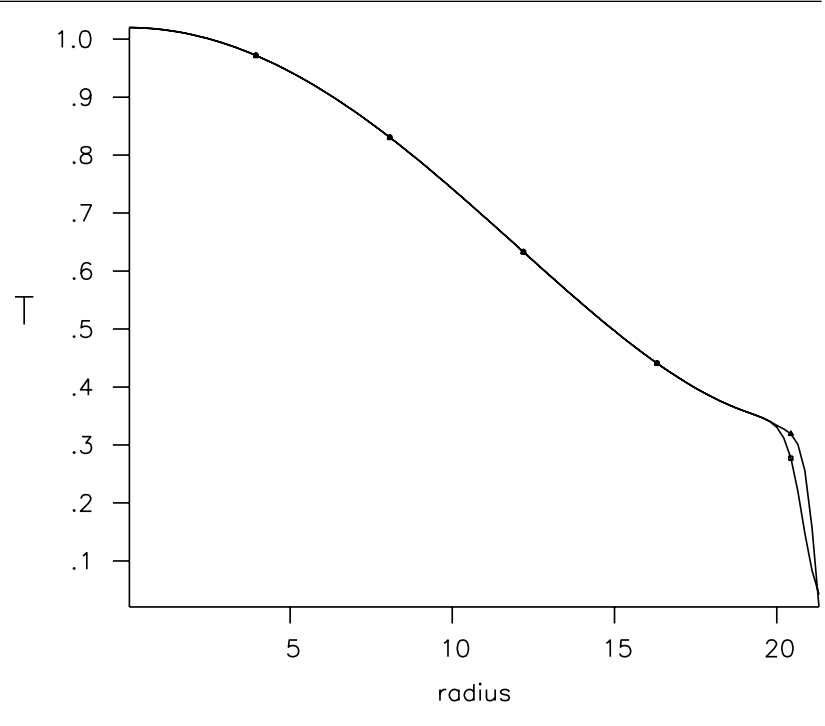

Figure 10. Ion temperature in ALCATOR [keV] versus $r$ [cm]. The lower curve is the experimental one and the upper belongs to the coefficient in equation (3) thus demonstrating the accurate approximation.

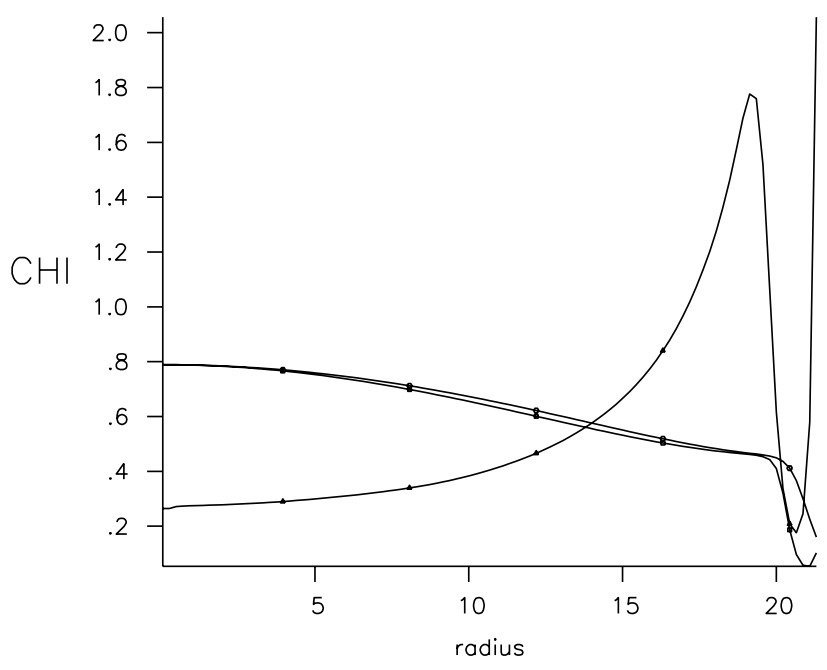

Figure 11. Temperature diffusivities $\left[\mathrm{m}^{2} \mathrm{~s}^{-1}\right] \chi_{\mathrm{tot}}=\chi_{\text {inv }}(\triangle) \chi_{\mathrm{sub}}$ $(\square), \chi_{\text {neo }}(O)$ versus radius $r[\mathrm{~cm}]$. At the inflection point $\chi_{\text {tot }}$ lies in between the neoclassical and the subneoclassical diffusivity, demonstrating that a subneoclassical state is reached.

(3) As distribution for the radiation losses the distribution of figure 2 is used. This distribution is normalized to $1 / 2$ of the Ohmic input power (400 kW) [24].

(4) As beam power deposition profile a Gaussian centred around $r=0$ with a half width $\delta_{\text {beam }}=20 \mathrm{~cm}$ is used.

Figure 12 displays the L-mode temperature profile together with the H-mode profile evoked by the divertor by reducing the neutral gas influx thus increasing the velocity shear; the toroidal velocity profile is shown in figure 13. As in ALCATOR we have a pronounced pedestal and the central velocity is $120 \mathrm{~km} \mathrm{~s}^{-1}$.

The pressure gradient is in the case of the profiles considered before beyond the ballooning-peeling limit given by $[16,17] \alpha_{\text {ball }} \alpha_{\text {peel }}>1$ with $\alpha_{\text {ball }}=\left(\beta q^{2} R / L_{\mathrm{p}}\right)$ and $\alpha_{\text {peel }}=1+\left(2.44(1+\eta) \delta_{\mathrm{R}}\right) /\left(\sqrt{\epsilon} L_{\mathrm{p}}\right), L_{\mathrm{p}}$ is the pressure gradient length, $\beta_{\mathrm{in}}$ is the local $\beta$ at the inflection point and $\delta_{\mathrm{R}}$ 


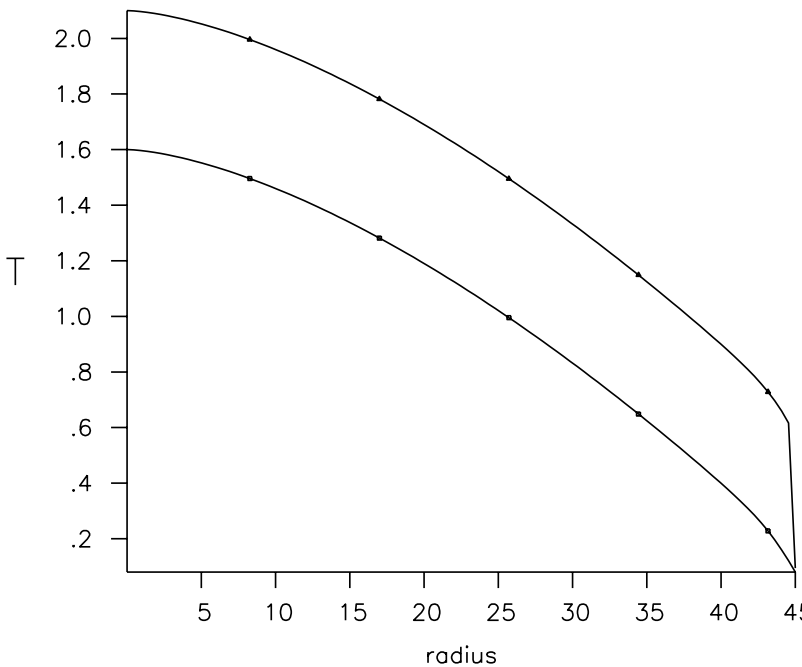

Figure 12. L- and H-mode temperatures in a medium size tokamak versus radius $r[\mathrm{~cm}]$. The $\mathrm{H}$-mode profile is obtained by inverting the L-mode profile and by using the thus obtained coefficient outside the pedestal region and by resorting to the subneoclassical dependence in the edge as in the case of ALCATOR.

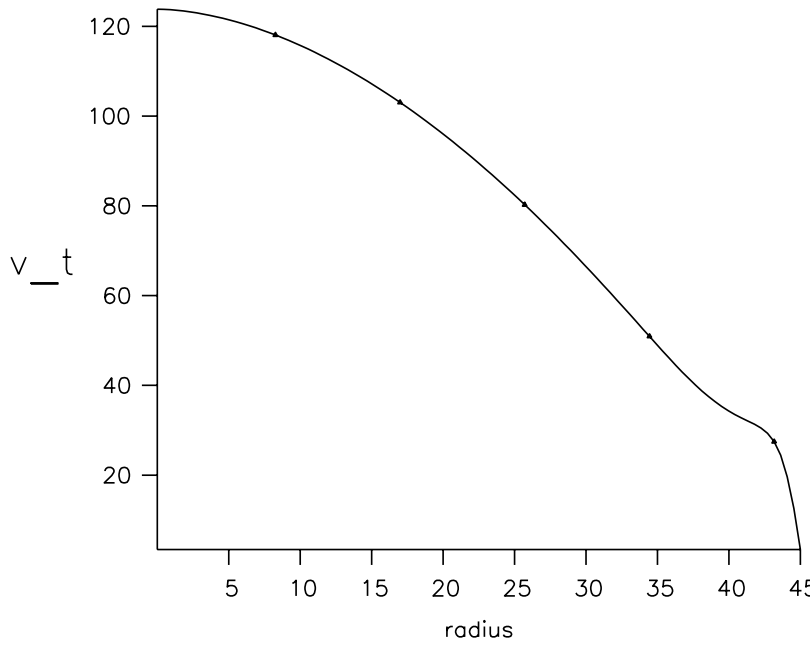

Figure 13. Toroidal velocity $\left[\mathrm{km} \mathrm{s}^{-1}\right]$ versus radius $r[\mathrm{~cm}]$ in the medium size tokamak in the case of the H-mode. The velocity profile has a pedestal as in ALCATOR.

is the length $\delta_{\mathrm{R}}=\left[R \rho_{\mathrm{s}}^{2} / 4\right]^{1 / 3}\left(\rho_{\mathrm{s}}=c_{\mathrm{s}} / \Omega_{\mathrm{i}}, c_{\mathrm{s}}=10^{4} \sqrt{T_{\mathrm{e}} / A_{\mathrm{i}}}\right)$. Since the common action of the ballooning and the peeling instability [17] are considered here to be the origin of the ELMs we take $\alpha_{\text {ball }} \alpha_{\text {peel }}>1$ as threshold for the ELM-excitation.

We mention that according to [18] the limit of the ballooning-peeling instability is increased from unity to $\alpha_{\text {field }}=1+\left(q R / \delta_{\mathrm{R}}\right)^{2}(\delta B / B)^{2} . \quad \delta B$ stands for the high $m$ Fourier component. This means a stabilizing action without changing the profile parameters. This seems to be confirmed by the DIII-D observations, where only weak changes in the profiles had been observed [15].

The ELMS are a danger for the divertor plates because the loading may exceed, e.g. in ITER, $45 \mathrm{MJ} \mathrm{m}^{-2} \mathrm{~s}^{-1}$. Therefore mitigation of ELMs by ergodizing the magnetic field in the edge [15] is considered here. Figure 14 displays the Poincaré plot (obtained with the Gourdon code [19]) in the case of a low perturbation current $\left(I_{\text {hel }}=1 \mathrm{kA}\right)$. The radial extension

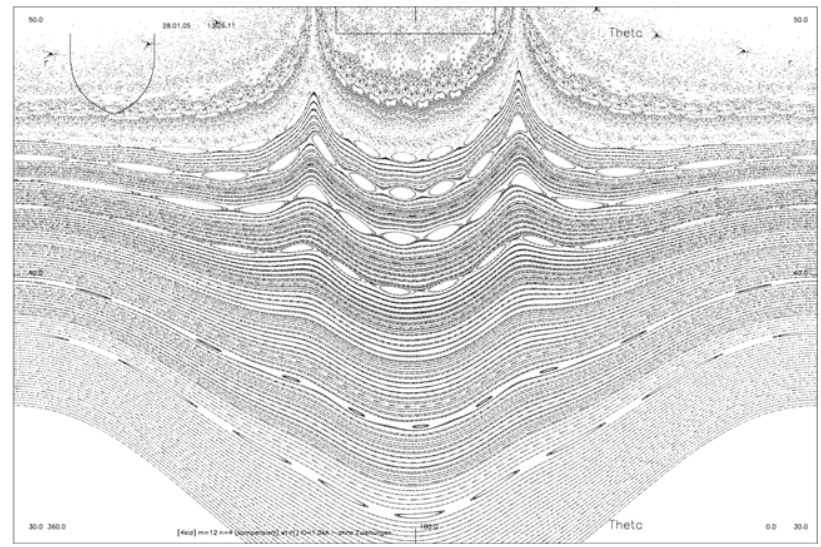

Figure 14. Poincaré plot of the separatrix region. The horizontal axis stands for the poloidal angle in $\left[0^{\circ}-360^{\circ}\right]$, the vertical axis for the radius in $[30-50 \mathrm{~cm}]$. The standard $12 / 4$ configuration of DED with $I_{\text {hel }}=1 \mathrm{kA}$ is used. The region around the separatrix is ergodized, whereas inside the separatrix KAM-surfaces with island chains appear.

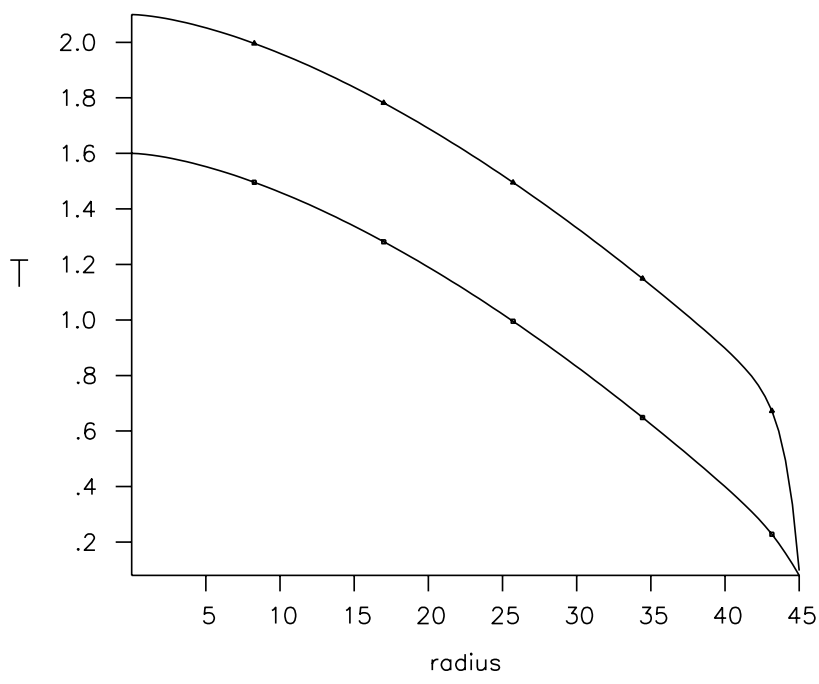

Figure 15. Ion temperature $T_{\mathrm{i}}[\mathrm{keV}]$ versus radius $r[\mathrm{~cm}]$ for the $\mathrm{L}$ -mode plasma and an H-mode plasma with an ergodized separatrix region. The same pedestal height as in figure 12 can be reached if the transition length of L-mode transport and $\mathrm{H}$-mode transport is broadened.

in figure 14 is given by $(r=30 \mathrm{~cm}<r<r=50 \mathrm{~cm})$. The DED-coils (in figure 16, in the $12 / 4$ configuration) are somewhat outside the depicted region at $\left(\theta_{1}=215^{\circ}>\right.$ $\left.\theta>\theta_{2}=145^{\circ}, r=51 \mathrm{~cm}\right)$. The separatrix region seems to be sufficiently ergodized, even at the low current $I_{\text {hel }}$, whereas inside the separatrix good confinement zones with small islands appear. This is compatible with [20] where it was shown that even small perturbing fields are sufficient to perturb the separatrix. We note that these results stay qualitatively the same if the X-points are pulled apart (e.g. to the top and bottom of the plasma (figure 18)).

According to the experiences at DIII-D [15], the confining edge region can be broadened without deteriorating the central confinement.

In figure 15 the confining zone is broadend from $\Delta=$ $1 \mathrm{~cm}$ to $\Delta=2.5 \mathrm{~cm}$ because the radial extension of the ergodized region at the separatrix in figure 15 is of the order 


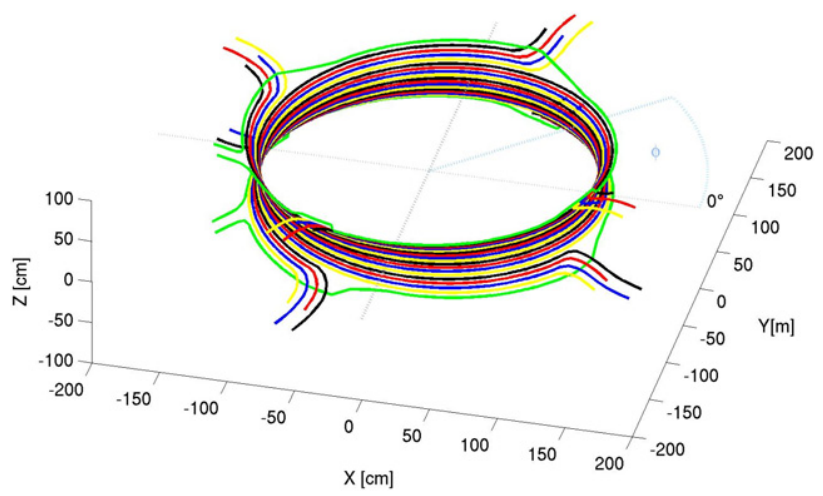

Figure 16. The drawing of the DED-coils shows that the winding consisting of 16 conductors with independent feeders can generate the modes $(m=12, n=4),(m=6, n=2),(m=3, n=1)$

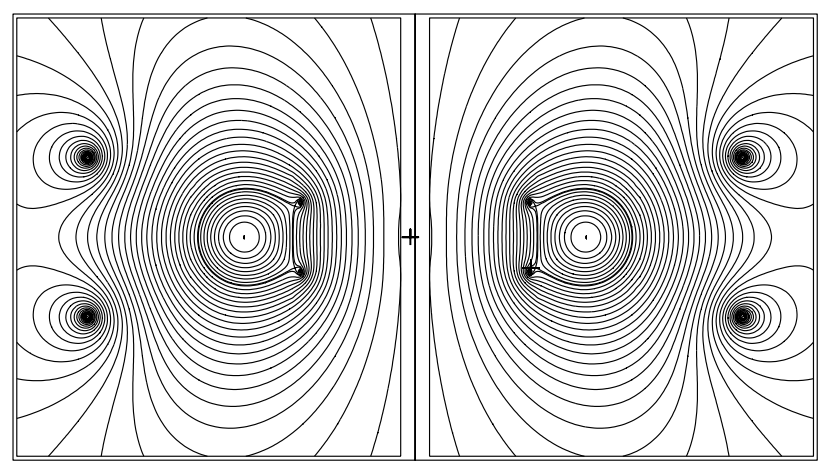

Figure 17. Figure 17 shows the equilibrium configuration used here for medium size tokamak. In contrast to other divertor configurations it has the X-points at the high field side, which may provide the possibility of meeting new physical conditions.

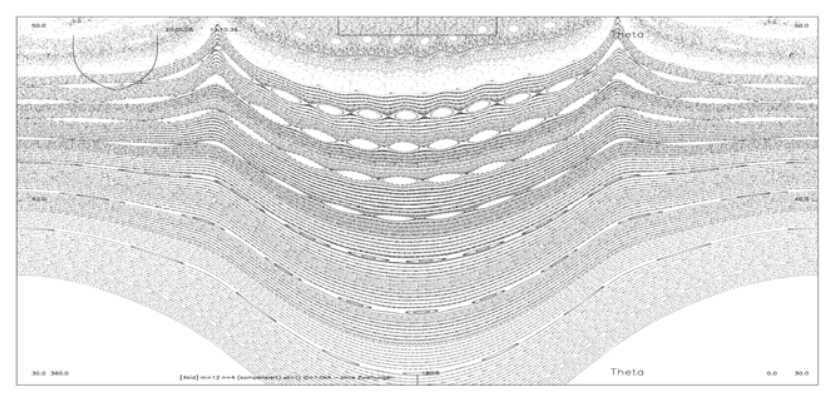

Figure 18. Poincaré plot of the separatrix region, analogous to figure 14. The horizontal axis stands for the poloidal angle in $\left[0-360^{\circ}\right]$, the vertical axis for the radius in $[30-50 \mathrm{~cm}]$. The divertor coils are now on the top and bottom of the plasma.

of $2 \mathrm{~cm}$. (The extension is measured from the inner island structures to the outer island structure, both neighbouring the unperturbed separatrix.) This pushes the product $\alpha_{\text {ball }} \alpha_{\text {peel }}$ below unity (this limit may be larger because of the factor $\alpha_{\text {field }}$ ). This radial dependence is obtained by changing the transition length from $\delta_{\text {trans }}=0.1 \mathrm{~cm}$ to $\delta_{\text {trans }}=0.65 \mathrm{~cm}$. $\delta_{\text {trans }}$ is the second parameter which must be adjusted to meet the pedestal height. The increase of $\delta_{\text {trans }}$ means that the L-mode transport properties partly 'intrude' into the pedestal region. The observations at DIII-D seem to confirm this [15].
We note, however, that because of the uncertainties, in particular concerning the width of the ergodized layer, the above estimates may be rather rough.

\section{Conclusions and discussion}

The inclusion of the braking term due to pressure anisotropization of [11] and the source term due to the $\vec{j} \times \vec{B}$ force density at the singular surface of [13] into the (revisited) neoclassical theory shows that at the singular surface the latter term dominates if the slip frequency is below the critical value whereas outside this surface NBI and viscosity losses are important.

The calculations show that there can be a strong impact of weak helical perturbations on the rotation velocity if the slip frequency is below its critical value given in [12]. If this condition is not fulfilled, the rotating fields must be considerably larger than the field required at $\omega=0$ to brake or accelerate the plasma rotation.

The importance of the slip frequency was underlined at JET [12]: due to the plasma rotation the stationary helical field did not penetrate into the singular surface and no braking was observed.

In spite of the locally very strong braking term (in the case $\omega=0$ ) it seems to be difficult to produce a strong gradient in the rotation field leading to an ITB, mainly because of the reduction of the maximum velocity at the plasma centre. However, a strong gain in the velocity gradient, possibly leading to an ITB, can be achieved by accelerating the plasma by ac-operation with a frequency of $10 \mathrm{kHz}$.

In a medium size tokamak, equipped with poloidal divertor, the plasma rotation is strongly enhanced due to the reduction of the neutral gas density at the last closed flux surface. As a consequence in a thin neoclassical sheath a temperature pedestal is built up. Combining the poloidal divertor with resonant ergodization coils, like the DED, provides the possibility of increasing the transport in the pedestal region without changing the height of the temperature pedestal. This procedure is currently under discussion as a means for ELM-mitigation which is an important issue for JET and ITER. Here, however, it is difficult to use resonant coil configurations like in TEXTOR.

\section{References}

[1] Stringer T.E. 1969 Phys. Rev. Lett. 22770

[2] Rogister A. 1994 Phys. Plasmas 1619

[3] Claassen H.A., Gerhauser H., Rogister A. and Yarim C. 2000 Phys. Plasmas 73699

[4] Nicolai A., Rogister A.L. and Daybelge U. 2002 Contrib. Plasma Phys. 42241

[5] Rogister A.L., Rice J.E., Nicolai A., Ince-Cushmann A., Gangadhara S. and Alcator C-Mod group 2002 Nucl. Fusion 421144

[6] Boivin R. 2002 private communication, General Atomics, San Diego, CA, USA

[7] Rogister A.L. 1999 Phys. Plasmas 6200

[8] Braginskii S.J. 1966 Transport processes in a plasma Rev. Plasma Phys. vol 1 (New York: Consultants Bureau) p 205

[9] Mikhailowski A.B. and Tsypin V.S. 1984 Beiträge zur Plasmaphysik 24335

[10] Tokar M.Z. et al 1999 Plasma Phys. Control. Fusion 41 B317 
[11] Lazzaro E. and Zanca P. 2003 Phys. Plasmas 102399

[12] Nicolai A., Daybelge U. and Yarim C. 2004 Nucl. Fusion 44 S93-107

[13] Fitzpatrick R. 1992 Theory of fusion plasmas Proc. Joint Varenna-Lausanne Int. Workshop (Varenna) p 147, Fitzpatrick R. 1995 Phys. Plasmas 21648

[14] Audenaerde K., Emmert G.A. and Gordinier M. 1978 University of Wisconsin Report UWFDM-259

[15] Evans T.E. et al 2004 Phys. Rev. Lett. 92 235003-1

[16] Wesson J. 1997 Tokamaks (Oxford: Clarendon Press)

[17] Connor J.W., Hastie B.J., Wilson H.R. and Miller R.J. 1998 Phys. Plasmas $\mathbf{5} 2687$

[18] Singh R. 2005 private communication, Institute for Plasma Research, Gandhinagar-382 428, India

[19] Gourdon C. 1970 Report IPP P12 483, Garching (translated by J.Eisert)
[20] Mel'nikov V.K. 1962 Sov. Phys._Dokl. 7502

[21] Nicolai A., Daybelge U. and Yarim C. 2004 Theory of fusion plasmas Proc. Joint Varenna-Lausanne Int. Workshop (Varenna) p 381

[22] Nicolai A., Daybelge U. and Yarim C. 2002 Transport analysis of H-mode plasma in Ohmic ALCATOR C-MOD discharges based on neoclassical theory Proc. 29th EPS

Conf. on Plasma Physics and Controlled Fusion (Montreux, Switzerland) Paper P 1.052

[23] Becoulet M., Thomas P., Nardon E. and Huysmans G. 2005 Comparison of DIII-D and possible JET and ITER ergodic coils designs 2nd Int. Workshop on Stochasticity in Fusion Plasmas (Jülich, Germany)

[24] von Hellermann M. 2004 private communication, Institut für Plasmaphysik, Forschungszentrum Jülich GmbH, Euratom Association, Trilateral Euregio-Cluster 